\title{
Phage-Displayed Peptides Selected to Bind Envelope Glycoprotein Show Antiviral Activity against Dengue Virus Serotype 2
}

\author{
Carolina de la Guardia, ${ }^{1,2}$ Mario Quijada, ${ }^{1}$ and Ricardo Lleonart ${ }^{1}$ \\ ${ }^{1}$ Center of Cellular and Molecular Biology of Diseases, Instituto de Investigaciones Científicas y Servicios de \\ Alta Tecnología (INDICASAT AIP), Building 219, Ciudad del Saber, Apartado 0843-01103, Panamá, Panama \\ ${ }^{2}$ Department of Biotechnology, Acharya Nagarjuna University, Guntur, India \\ Correspondence should be addressed to Ricardo Lleonart; rlleonart@indicasat.org.pa
}

Received 25 May 2017; Accepted 26 July 2017; Published 10 September 2017

Academic Editor: Gary S. Hayward

Copyright @ 2017 Carolina de la Guardia et al. This is an open access article distributed under the Creative Commons Attribution License, which permits unrestricted use, distribution, and reproduction in any medium, provided the original work is properly cited.

\begin{abstract}
Dengue virus is a growing public health threat that affects hundreds of million peoples every year and leave huge economic and social damage. The virus is transmitted by mosquitoes and the incidence of the disease is increasing, among other causes, due to the geographical expansion of the vector's range and the lack of effectiveness in public health interventions in most prevalent countries. So far, no highly effective vaccine or antiviral has been developed for this virus. Here we employed phage display technology to identify peptides able to block the DENV2. A random peptide library presented in M13 phages was screened with recombinant dengue envelope and its fragment domain III. After four rounds of panning, several binding peptides were identified, synthesized, and tested against the virus. Three peptides were able to block the infectivity of the virus while not being toxic to the target cells. Blind docking simulations were done to investigate the possible mode of binding, showing that all peptides appear to bind domain III of the protein and may be mostly stabilized by hydrophobic interactions. These results are relevant to the development of novel therapeutics against this important virus.
\end{abstract}

\section{Introduction}

Dengue virus is a growing public health problem worldwide as about 390 million people get infected annually and almost 96 million people develop clinical manifestations of the disease [1]. Other authors estimate that about 3.9 billion people from 128 countries share the risk of infection with this virus [2]. Dengue virus can produce a wide spectrum of clinical presentations, from asymptomatic or mild manifestation to a more severe life threating manifestation, known as dengue hemorrhagic fever (DHF) and dengue shock syndrome (DSS). Severe dengue can be deadly, especially in children, due to plasma leaking, respiratory distress, edema, severe bleeding, and organ impairment [3].

This virus is a Flavivirus that is transmitted by mosquitoes of the genus Aedes. The incidence of dengue infections has increased due to the spread of these mosquitoes into new regions [4]. DENV are present as 4 serotypes (DENV1, DENV2, DENV3, and DENV4) circulating in most endemic countries [5]. Dengue fever is considered the most rapidly growing mosquito-transmitted disease worldwide [6].

The dengue virion has an icosahedral symmetry, with diameter between $500 \AA$ and $600 \AA$ [7]. The viral genome consists of $11 \mathrm{Kbp}$ single positive stranded RNA coding for a single polyprotein. The polyprotein is cleaved in the cytoplasm into several structural and nonstructural polypeptides [8]. The structural proteins include the capsid (C), premembrane $(\operatorname{PrM}) /$ membrane $(\mathrm{M})$, and envelope glycoprotein (E), which are involved in the formation of the viral particle. The nonstructural proteins (NS1, NS2a, NS2b, NS3, NS4a, NS4b, and NS5) are responsible for the viral replication, assembly, and immune response escape [8].

E glycoprotein is the most important molecule during the viral entry process as it appears to be responsible for 
receptor recognition and attachment to the cell surface, triggering the clathrin mediated endocytosis and the subsequent fusion of viral and cellular membranes. This protein can interact with diverse cellular molecules; therefore, it is an ideal target for the development of new antivirals [9-12]. E glycoprotein is formed by three domains, plus a membrane proximal stem and a transmembrane anchor [13-15]. Domain I is formed by eight $\beta$-strand barrels containing two insertion loops. Domain II contains hydrophobic sequences that are conserved among all flaviviruses. These hydrophobic sequences, also known as fusion peptides, are responsible for the insertion of the rearranged $\mathrm{E}$ trimer into the cellular membrane during fusion $[9,14-16]$. Domain III (DIII) is an immunoglobulin-like carboxyterminal domain, responsible for the initial cellular receptor binding [17]. Additionally, the DIII is the main target for neutralizing antibodies [18].

Due to the complexities of the immune response and the pathology generated by this virus, particularly during subsequent infections, the development of vaccines has been slow and there is only one vaccine that has been registered, which is still in the early stages of testing in several territories $[19,20]$.

Currently, there is no approved antiviral for the treatment of dengue infection. It is known that the duration of the viremia is short in dengue patients. However, since high viremia has been related to a severe onset of the disease, the use of antivirals at early stages of the disease may block the progression of the disease and accelerate the recovery of patients. Here, we proposed the use of dengue virus envelope (E) glycoprotein as a target to search for peptides that could interfere with the first step of the infection process. We selected the DENV-2 E glycoprotein and the DIII as targets for the screening random peptides displayed on M13 bacteriophages.

Phage display technology has been used in past years to identify peptides with specific binding activities to a variety of targets. This technique has been very useful to identify mimotopes, novel antivirals [21], peptidomimetic drugs [22], and many other applications.

Here we show that the DENV2 E glycoprotein, as well as domain III (DIII), is useful target for the identification of phage-displayed peptides with potential as novel antivirals. Here we describe three peptides that inhibit the viral infectivity and are not cytotoxic to the permissive cells. Fully blind docking simulations with these peptides suggest binding sites at domain III of the envelope protein, stabilized by predominant hydrophobic interactions. These findings open new possibilities to optimize and refine the design of new peptidic inhibitors of infection by this virus.

\section{Materials and Methods}

2.1. Cells, Viruses, and Peptides. Vero cells (CCL-81, ATCC, USA) were used for dengue 2 virus propagation, plaque formation assays, and cytotoxicity assays. These cells were grown in MEM with $10 \%$ FCS and $50 \mu \mathrm{g} / \mathrm{ml}$ gentamicin, at $37^{\circ} \mathrm{C}$ and $5 \% \mathrm{CO}_{2}$. Dengue virus, serotype 2 , was a kind gift from Department of Research, Instituto Conmemorativo
Gorgas de Estudios de la Salud (ICEGS), Panamá. Heptapeptide random library was obtained from New England Biolabs (USA). Synthetic peptides were obtained from Genscript (USA). Full DENV2 E glycoprotein (Cat. number Den034) was obtained from ProSpec-Tany TechnoGene (Israel). Tissue culture, molecular biology grade, and general reagents were from Nunc and Sigma-Aldrich (USA).

2.2. Recombinant Protein Expression. Sequence coding for DENV2 E DIII (aa 289 to 405) was obtained from GenBank (complete genome NCBI Reference Sequence: NC_001474.2) and optimized for expression in Escherichia coli using an online tool (GeneOptimizer, Geneart). The optimized sequence was synthesized (IDT) and subcloned into NdeIXhoI restriction sites of the expression plasmid pET-30b(+) (Novagen) using standard recombinant DNA techniques. This plasmid directs the inducible production of the DIII plus the amino acids Leu and Glu and a 6xHis tail at the C-terminal. This fragment of the $\mathrm{E}$ protein contains 125 amino acids and an approximate molecular weight of $14.2 \mathrm{kDa}$. The expression plasmid was verified by sequencing and transformed into E. coli strain BL21 (DE3) for expression. Single colonies were cultured in LB medium containing antibiotic at $37^{\circ} \mathrm{C}$ and $250 \mathrm{rpm}$ and induced with $0.5 \mathrm{mM}$ IPTG when $\mathrm{OD}_{600}$ reached 0.6. After 4 hours of induction cells were collected by centrifugation and kept at $-20^{\circ} \mathrm{C}$ until purification. As expression of the recombinant protein was mostly at the insoluble fraction, further purification was done including inclusion body isolation, solubilization, and affinity purification in denaturing conditions, refolding, and dialysis.

2.3. Purification of Recombinant DIII. Frozen pellet from induced culture was suspended in cold lysis buffer $(10 \mathrm{mM}$ Tris- $\mathrm{HCl} \mathrm{pH} 7.5,5 \mathrm{mM}$ benzamidine-HCl, $5 \mathrm{mM}$ EDTA, $5 \mathrm{mM}$ DTT, $0.3 \mathrm{mg} / \mathrm{ml}$ lysozyme, and $1 \mathrm{mM}$ PMSF) and lysed by sonication on ice. The inclusion bodies were recovered by centrifugation, washed with $50 \mathrm{mM}$ phosphate buffer, $5 \mathrm{mM}$ EDTA, $200 \mathrm{mM} \mathrm{NaCl}, 0.5 \mathrm{M}$ urea, and $1 \%$ Triton X-100, recovered as before, washed with $50 \mathrm{mM}$ phosphate buffer, $1 \mathrm{mM}$ EDTA, and $1 \mathrm{M} \mathrm{NaCl}$, and solubilized in denaturing buffer (10 mM Tris- $\mathrm{HCl}$ pH 8.0, $100 \mathrm{mM} \mathrm{NaH}{ }_{2} \mathrm{PO}_{4}, 100 \mathrm{mM}$ $\mathrm{NaCl}$, and $6 \mathrm{M} \mathrm{GuHCl}$ ). The solubilized inclusion bodies were purified using a Ni-NTA superflow cartridge (Qiagen). Following loading and washing with buffer $\mathrm{C}(6 \mathrm{M} \mathrm{GuHCl}$,

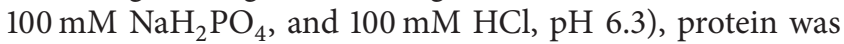
eluted with buffer $\mathrm{E}\left(6 \mathrm{M} \mathrm{GuHCl}, 100 \mathrm{mM} \mathrm{NaH}_{2} \mathrm{PO}_{4}\right.$, and $100 \mathrm{mM} \mathrm{HCl}, \mathrm{pH}$ 4.5). Attempts to dialyze against PBS resulted in protein precipitation; therefore we tested several refolding conditions using a Protein Refolding kit (Pierce, USA). Finally, refolding was done by dilution into refolding buffer RB7 (1 mM GSH, $1 \mathrm{mM}$ GSSH, $1 \mathrm{mM}$ EDTA, $1.1 \mathrm{M}$ $\mathrm{GuHCl}, 55 \mathrm{mM}$ Tris- $\mathrm{HCl}, \mathrm{pH} 8.0,21 \mathrm{mM} \mathrm{NaCl}$, and $0.88 \mathrm{mM}$ $\mathrm{KCl})$. The refolded protein was dialyzed against TBS, filter sterilized, and quantitated by BCA method.

2.4. In Vitro Virus Blocking Assay to Test Protein Refolding. Aliquots of refolded recombinant DIII, obtained from several refolding conditions, were first visually checked for 
aggregation. Those not showing aggregation were then tested in virus infection blocking experiments, to check for their ability to bind cellular receptors and inhibit these first steps in viral infection. Vero cells were plated in 96-well plates at $10^{4}$ cells per well in complete medium (MEM, $10 \%$ FCS, and $50 \mu \mathrm{g} / \mathrm{ml}$ gentamicin). On the next day, cells were washed with PBS and incubated with the refolded protein $(100 \mu \mathrm{l}$, $50 \mu \mathrm{g} / \mathrm{ml}$ ) in maintenance medium (MEM, 1\% FCS, and gentamicin) for $30 \mathrm{~min}$ at $37^{\circ} \mathrm{C}$. Then cells were washed and incubated with DENV2 (MOI $=3,50 \mu \mathrm{l})$, containing the refolded protein at the same concentration, for $1 \mathrm{~h}$ at $37^{\circ} \mathrm{C}$ in maintenance medium. Cells were again washed, replenished with maintenance medium, and incubated at $37^{\circ} \mathrm{C}, 5 \% \mathrm{CO}_{2}$. After 5 days, cytopathic effect was quantified using chemiluminescent based ATP detection (CellTiterGlo ${ }^{\circledR}$ cell viability assay, Promega).

2.5. Biopanning. Phage display was performed using a Ph.D.-7 Phage Display Peptide Library according to the manufacturer's instructions. Four rounds of panning were done using two targets, the full DENV2 envelope, or the recombinant DIII obtained as described above. For each panning step, several wells in ninety-six-well plates were coated overnight at $4^{\circ} \mathrm{C}$ and then blocked with $400 \mu \mathrm{l}$ of $0.1 \mathrm{M}$ $\mathrm{NaHCO}_{3}\left(\mathrm{pH} \mathrm{8.6)}\right.$ and $5 \mathrm{mg} / \mathrm{ml} \mathrm{BSA}$ for $1 \mathrm{~h}$ at $4^{\circ} \mathrm{C}$. After six washes with TBST (TBS, $0.1 \%$ Tween 20 ), $100 \mu$ l of phages in TBST, containing $10^{11}$ pfu were allowed to bind for $1 \mathrm{~h}$ at RT. Then wells were washed ten times with TBST and phages eluted with $0.2 \mathrm{M}$ glycine- $\mathrm{HCl} \mathrm{pH} 2.2,1 \mathrm{mg} / \mathrm{ml}$ BSA. After neutralization with Tris $\mathrm{pH}$ 9.1, phages were titered and bulk amplified in $E$. coli for next round. In order to increase the stringency of the selection, successive rounds were done increasing the concentration of Tween-20 during washes and reducing the amount of target fixed to the solid phase. After the fourth round, eluted phages were plated, picked, and propagated for subsequent phage-ELISA and sequencing.

2.6. Phage-ELISA. DENV2 E, DIII, and BSA were used to coat 96-well microtiter plate (MaxiSorp, Nunc) overnight at $4^{\circ} \mathrm{C}$. Plates were blocked with TBS-BSA, washed with TBST, and incubated with $10^{12}$ pfu of individual phages. After washing, HRP-conjugated anti-M13 monoclonal antibody (GE Healthcare, USA) was added, incubated, and washed. Color was developed by adding TMB substrate solution for $10 \mathrm{~min}$ and stopped with $1 \mathrm{~N} \mathrm{HCl}$. Plates were read at $405 \mathrm{~nm}$. Phage clones with the best target-to-background signal ratio were selected for DNA sequencing. The DNA insert was determined by cycle sequencing using a 96-gIII primer as recommended by manufacturer. Sequence logos and residue coloring was done as implemented in JalView v.2.10.1 [23]. Coloring was following Zappo scheme, where residues are colored according to their physicochemical properties as follows: pink, aliphatic/hydrophobic; orange, aromatic; red, positive; green, negative; blue, hydrophilic; magenta, proline/glycine; and yellow, cysteine.

2.7. Viral Plaque Reduction Assay. Vero cells were seeded $\left(2 \times 10^{5}\right.$ cells per well $)$ in 6 -well plates and incubated overnight. The peptides were prepared at several dilutions and mixed with 100 pfu of DENV2, for a final volume of $150 \mu \mathrm{l}$. Mixtures were incubated at RT for $1 \mathrm{~h}$ and then added to cell monolayers and incubated $1 \mathrm{~h}$ at $37^{\circ} \mathrm{C}$ for adsorption. Then inoculum was removed and cells were washed and overlaid with maintenance medium with $1 \%$ methylcellulose and incubated for 5 days. Then medium was aspirated; cells were fixed with $10 \%$ formaldehyde and stained with $1 \%$ crystal violet. The viral plaques were visually counted and values from experimental groups compared to virus alone, nontreated controls.

2.8. Peptide Cytotoxicity Assay. Peptides were incubated with cells exactly as described in the viral plaque reduction assay ( $1 \mathrm{~h}$ for $37^{\circ} \mathrm{C}$ ) and then washed away and cells refed with maintenance medium and incubated at $37^{\circ} \mathrm{C}$ for $24 \mathrm{~h}$. Then cytotoxicity was estimated by measuring cellular ATP using luminescence as described by the manufacturer of the CellTiter-Glo ${ }^{\circledR}$ cell viability assay. Viability was estimated by comparing readings of peptide-treated cells with those of nontreated cells.

2.9. Peptide-Protein Docking Simulations. The proteins structures used for the peptide-docking analysis were either obtained from the database of protein structures (in the case of the DENV envelope protein, PDB 1OAN) or obtained in our lab by homology modeling (for DIII). Sequence corresponding to the expressed protein was used to obtain the structure by homology modeling using I-TASSER web server [24]. The best model predicted by I-TASSER presented good predictive value $(C$-score $=0.77)$ that was structurally closely related to several viral envelope glycoproteins in the PDB database. To study the probable binding mode of the active peptides, we performed computational docking analysis using CABS-dock web server with the default parameters except for the increase of simulation cycles to 200 [25]. The best poses, ranked according to trajectory characteristics, were further analyzed to describe intermolecular interactions using $\operatorname{LigPlot}^{+}[26]$.

2.10. Statistical Analysis. Where indicated, medians were compared between the nontreated control and all other groups using Kruskal-Wallis with Dunn's multiple comparison test, as implemented in GraphPad Prism software.

\section{Results and Discussion}

3.1. Expression, Purification, and Refolding of Recombinant DENV2 Envelope Domain III. The dengue virus envelope is a class II virus fusion protein. This protein is a $\beta$ strand rich, elongated molecule with three ectodomains, the centrally located DI, the apical DII which bears the fusion loop, and the Ig-like DIII, which is connected to the short, stem, and transmembrane C-terminus [14, 27]. DIII undergoes drastic repositioning during transition to the fusogenic conformation of the E protein, leading to rearrangement from dimeric to trimeric form $[15,28]$. DENV E domain III forms an Ig-like $\beta$-barrel structure that is stabilized by one disulfide bridge. 


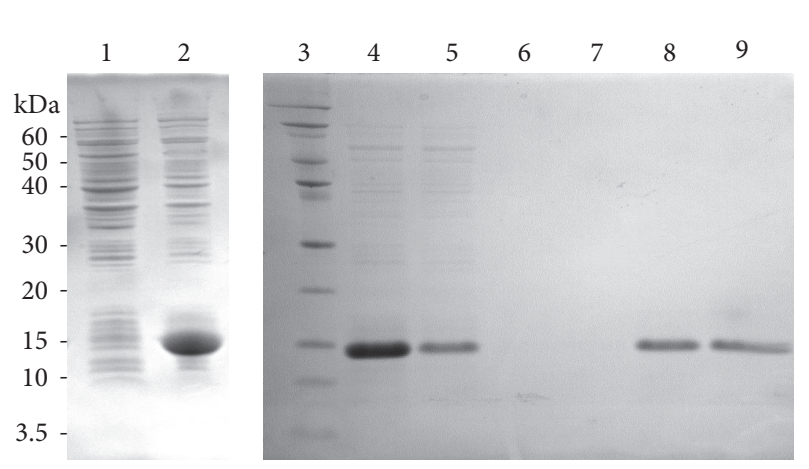

(a)

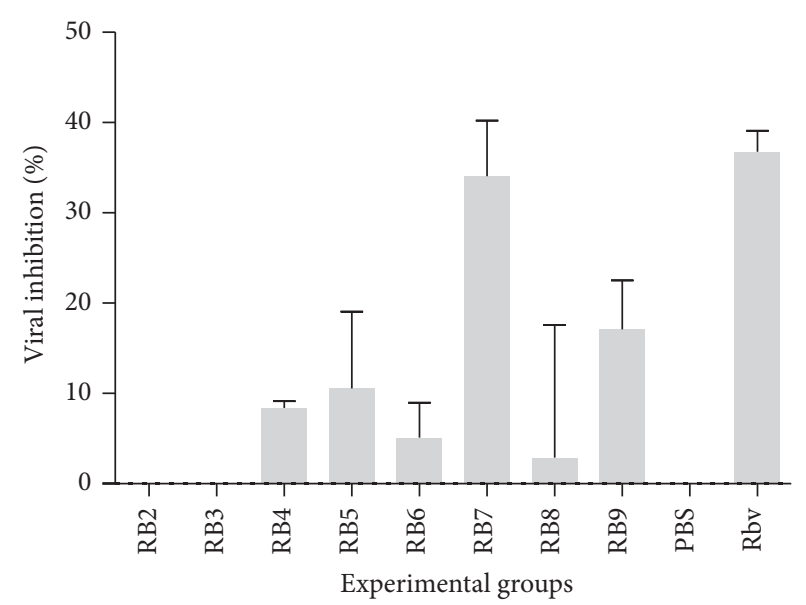

(b)

FIGURE 1: Cloning, expression in E. coli, and purification of codon optimized, dengue 2 envelope domain III. (a) SDS-PAGE showing several steps of protein expression and purification. 1, total lysate of cells before induction; 2, total lysate of cell after IPTG induction; 3, protein molecular weight standards; 4, solubilized inclusion bodies before loading into Ni-NTA column; 5, column flow-through; 6-7, two successive washes; 8-9, two successive elution. (b) Activity of purified and refolded recombinant domain III against DENV2 in Vero cells. Protein was refolded using several refolding conditions (RB1 to RB9) before dialysis and filtration for testing against the virus, in parallel with virus alone and ribavirin as a control. Data is presented as the mean and SD of two independent experiments. Condition RB1 was not used in this experiment as protein consistently precipitated.

The DIII is a key part of the envelope, as it is the receptor binding domain [13, 29]. It is also very antigenic, and antibodies to this domain are able to efficiently neutralize virus infection $[18,30]$. Taking into account the important role of the envelope protein, as well as its domain III, we decided to search for peptides with binding activity to this protein, in an attempt to find novel molecules able to impair the infection process. For this purpose, we selected phage display as a robust methodology that allows the rapid screening and selection of millions of peptidic variants for binding capacity to a variety of targets. As the full envelope glycoprotein is a large protein that may present many possible nonproductive binding sites, we chose also to screen the random peptide library with domain III obtained in our lab.

The region coding for domain III of the envelope protein of DENV2 was obtained as a synthetic fragment from a commercial source after codon optimization for $E$. coli expression. This fragment was subcloned into the $E$. coli expression vector pET-30b under the control of a T7 promoter and fused to a 6xHis tail at the C-terminal. After IPTG induction, E. coli cultures showed high level of expression of the recombinant protein (Figure 1(a)), accounting for more than $50 \%$ of total protein, as judged from densitometric analysis of Coomassie stained SDS-PAGE gels (data not shown). As the recombinant protein was mainly present in insoluble aggregates, the purification protocol included an isolation and wash of inclusion bodies, followed by an affinity purification by affinity Ni-NTA chromatography under denaturing conditions. The affinity purification procedure implemented here allowed a high purity preparation of DIII (Figure 1(a)).
Several attempts to refold this protein by dilution into PBS failed due to precipitation; therefore the refolding conditions for this protein were further explored using several experimental conditions. These tests included varying concentrations of refolding agents and additives, such as L-arginine, reduced and oxidized glutathione, and/or polyethylene glycol [31]. It has been shown that the dengue envelope DIII may act as a dominant negative inhibitor of the infection process [10, 32-35]; therefore we decided to use this criterion to assess the functionality of the refolded DIII. After the refolding tests, each protein preparation was dialyzed against TBS, filter sterilized, and tested in a DENV2 virus binding blocking assay in Vero cells. We found that recombinant protein refolded in buffer RB7 (1.1 M guanidine, $55 \mathrm{mM}$ Tris- $\mathrm{HCl}$, $\mathrm{pH}$ 8.2, $21 \mathrm{mM} \mathrm{NaCl}, 0.88 \mathrm{mM} \mathrm{KCl}, 1 \mathrm{mM} \mathrm{GSH}$, and $1 \mathrm{mM}$ EDTA) showed a significant inhibition of the infection (Figure 1(b)) while there were no signs of cytotoxicity (data not shown), suggesting that the protein was folded and functional. This procedure was then used at a higher scale to isolate the recombinant EDIII for the biopanning and further steps.

\subsection{Selection of Phage-Displayed Envelope Binding Peptides.} In order to select for peptidic binders to both targets, we used a random library of heptapeptides presented in the M13 phage pIII protein. These phages would contain 1 to 5 copies of the peptides per capsid, theoretically allowing for the selection of higher affinity binders. As baits for the panning procedure, two sources of proteins were used, (1) a recombinant, insectexpressed DENV2 envelope and (2) recombinant, E. coli expressed domain III. The panning procedure involved four rounds of phage selection on solid phase-bound protein 


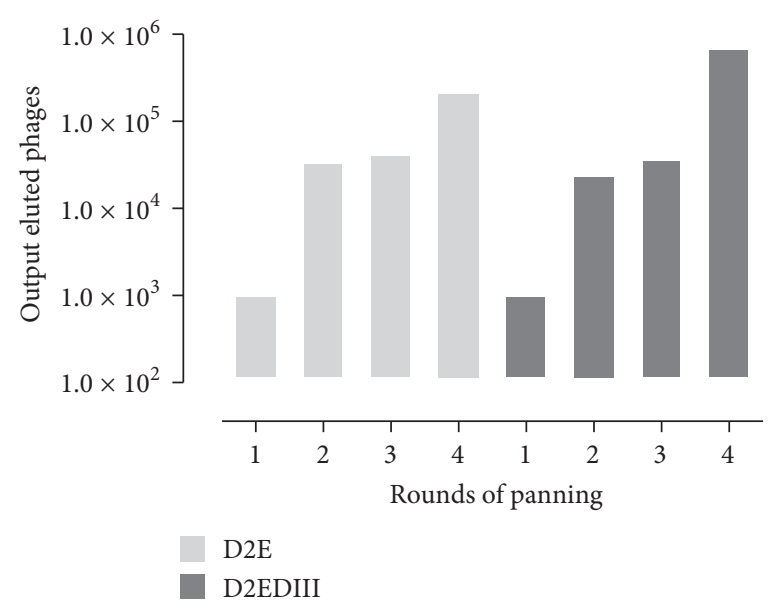

FIGURE 2: Eluted phages were enriched in successive rounds or panning. During each panning, $10^{11}$ input phages were incubated with the target proteins (full DENV2 envelope or domain III) and eluted as described in Materials and Methods. The eluted phages were neutralized and titer was estimated. Data is presented as the absolute number of eluted phages at each step.

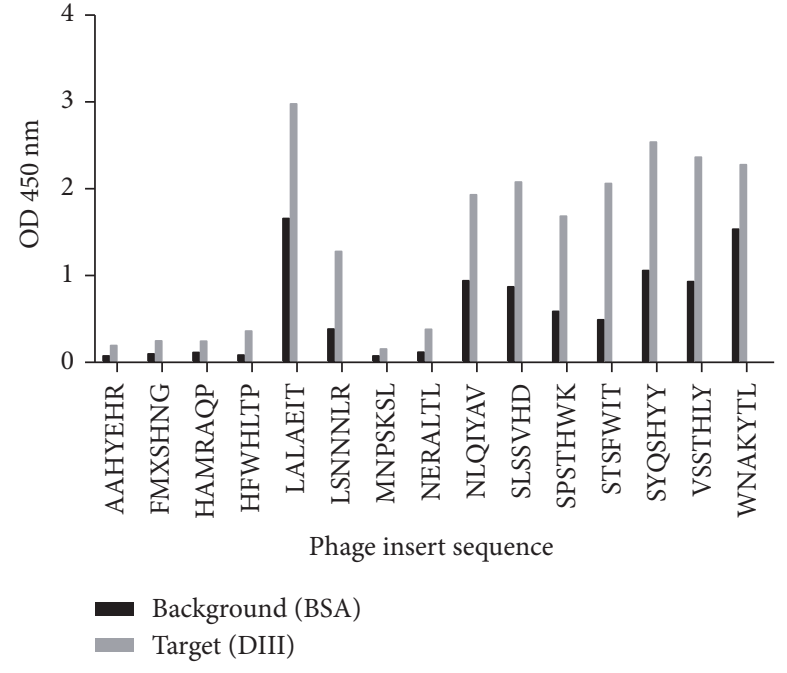

(a)

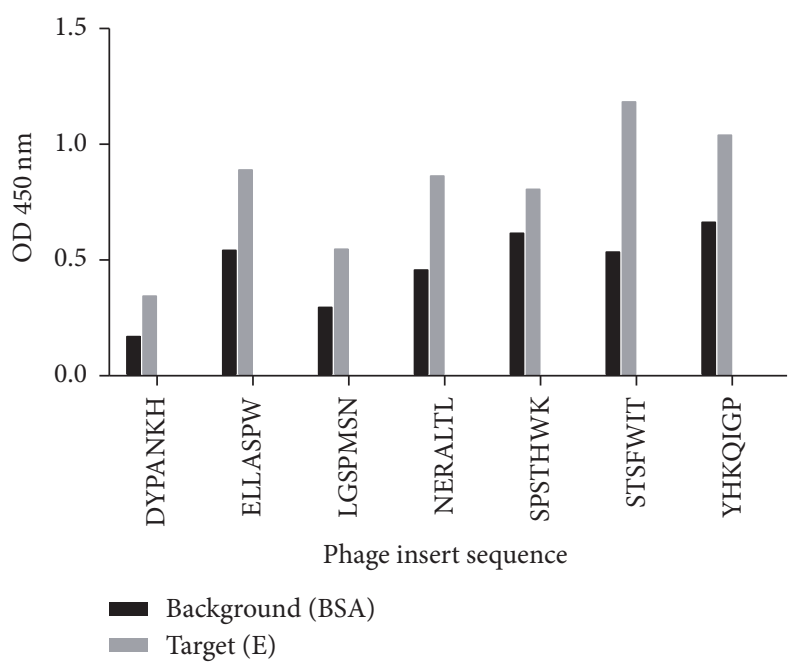

(b)

Figure 3: In vitro binding of selected clones as shown by phage-ELISA. Absorbance values by ELISA when phages are incubated in the presence of solid phase-bound specific ligand (target) or unspecific ligand (bovine serum albumin, as background). The binding of phages was revealed by anti-M13 antibody. (a) Results of phage-ELISA using E domain III as target. (b) Results obtained when using full E glycoprotein as target.

and subsequent elution and amplification. During panning progression against both proteins, the amounts of eluted phages increase stepwise (Figure 2), suggesting an effective enrichment of particular phages. After final round, 24 phages were randomly selected from each panning scheme, propagated, and purified to test their binding ability to the respective bait by phage-ELISA.

The analysis of eluted phages by ELISA showed that many appear to bind in a specific manner, indicated by absorbance values higher for the target as compared to those against the background (BSA) (Figure 3). Out of 24 randomly picked phages from each panning, 12 were confirmed for the full $\mathrm{E}$ glycoprotein and 21 were positive for the E domain III fragment. This behavior was shown by phages bearing the peptides STSFWIT, NERALTL, ELLASPW, SPSTHWK, LALAEIT, NLQIYAV, and SLSSVHD. Some other clones did not show good target-to-background signal ratio and were not used for sequencing or subsequent analyses. This result is not unexpected, as panning procedure may yield artifact binding phages which later do not bind well in the context of the ELISA.

The phage-ELISA positive phages, 33 in total, were submitted to DNA sequencing of the randomized region. DNA sequencing revealed that binding phages carried peptides with some common features, with similar consensus sequences for the envelope glycoprotein $(\mathrm{SxSAxxx})$ and for the domain III protein (SxSxHTL) (Table 1, Figure 4). The corresponding peptides had abundance of negatively charged 


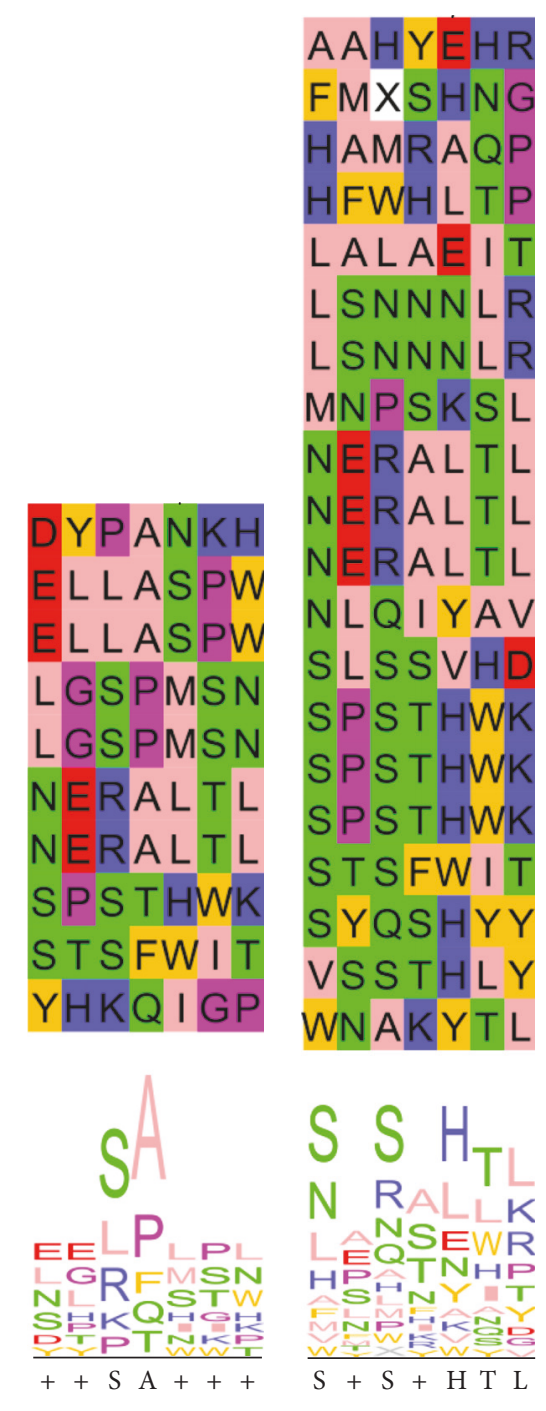

(a)

(b)

FIGURE 4: Peptide sequences resulting from binder phages confirmed by phage-ELISA. (a) Peptides resulting from panning on full DENV2 envelope. (b) Peptides resulting from panning on recombinant DENV2 envelope domain III. Sequence logos and consensus sequence, as calculated using JalView, are depicted below each list of peptides. Coloring of residues and sequence logos as per Zappo scheme, where residues are colored according to their physicochemical properties as follows: pink, aliphatic/hydrophobic; orange, aromatic; red, positive; green, negative; blue, hydrophilic; magenta, proline/glycine; and yellow, cysteine.

residues, intercalated with positively charged, hydrophilic, and aliphatic/hydrophobic residues (Figure 4). The resulting peptide sequences are consistent with one of the proteins (DIII) being a smaller portion of the other (E), as the consensus sequences share some resemblance, and the consensus for peptides selected against DIII appears to be more defined. Interestingly, several phages bearing the same peptide were selected with both proteins and appeared to be repeated in the final round elution (Table 1). The fact that some phages were observed several times is also congruent with a successful enrichment of specific binders during the panning steps.

Some of the binding phages shown here carried peptide sequences already reported at the biopanning data bank, a repository that, at the moment of writing this paper, had more than 23,700 sequences appearing in biopanning data [36]. The peptides SPSTHWK and WNAKYTL were also previously reported to bind crystalline $\mathrm{Ni3B}$ nanoparticles [37]. Also, the peptide NERALTL appeared twice in the database, with binding activity to epoxy covered surfaces [38] and to the fusion protein of the infectious salmon anemia virus [39]. Additionally, the peptide LSNNNLR was previously reported to bind poly(dimethylsiloxane) [40].

3.3. Activity of Selected Peptides and Possible Mode of Binding. Peptides from ELISA verified binding phages were synthesized containing the additional Gly-Gly-Gly-Ser spacer at the C-terminal and amidation of the carboxylate to block the negative charge, as suggested by the manufacturer to mimic 
TABLE 1: Peptide sequences at the randomized region and frequency of appearance of bearing phages.

\begin{tabular}{|c|c|c|c|}
\hline \multicolumn{2}{|c|}{ Target: envelope } & \multicolumn{2}{|c|}{ Target: domain III } \\
\hline Peptide & Frequency (\%) & Peptide & Frequency (\%) \\
\hline DYPANKH & 10 & AAHYEHR & 5 \\
\hline ELLASPW & 20 & FMXSHNG & 5 \\
\hline LGSPMSN & 20 & HAMRAQP & 5 \\
\hline NERALTL & 20 & HFWHLTP & 5 \\
\hline SPSTHWK & 10 & LALAEIT & 5 \\
\hline STSFWIT & 10 & LSNNNLR & 10 \\
\hline \multirow[t]{9}{*}{ YHKQIGP } & 10 & MNPSKSL & 5 \\
\hline & & NERALTL & 15 \\
\hline & & NLQIYAV & 5 \\
\hline & & SLSSVHD & 5 \\
\hline & & SPSTHWK & 15 \\
\hline & & STSFWIT & 5 \\
\hline & & SYQSHYY & 5 \\
\hline & & VSSTHLY & 5 \\
\hline & & WNAKYTL & 5 \\
\hline
\end{tabular}
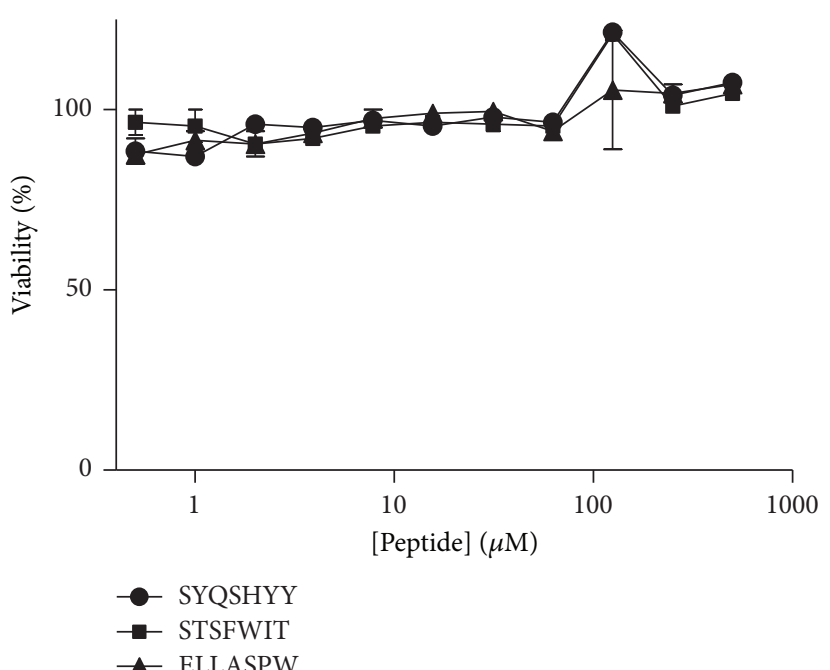

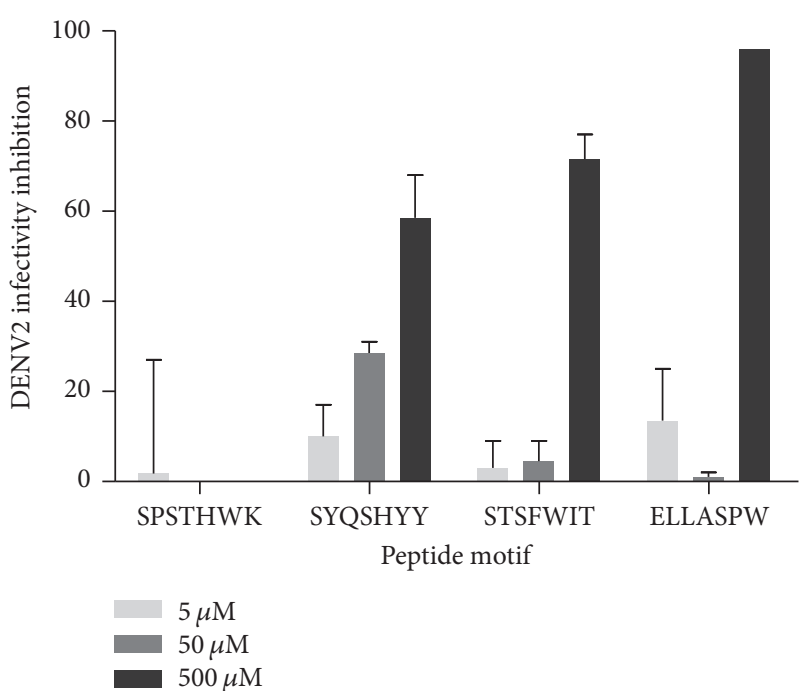

(b)

Figure 5: Biological activities of active peptides in Vero cells. (a) Cytotoxicity of active peptides. Cells were incubated with increasing concentrations of the peptide for $1 \mathrm{~h}$ and washed away and cells incubated in maintenance medium for 24 . Then cytotoxicity was estimated from ATP levels using luminescence. The viability was estimated by comparing with nontreated cells. Data is presented as means and standard deviations of two independent experiments. Medians were compared between the nontreated control and all other groups using KruskalWallis with Dunn's multiple comparison test. (b) DENV2 infectivity inhibition by peptides, as shown in a plaque reduction assay. The reduction in the number of plaques was estimated by comparison with virus-infected cells alone. The peptide SPSTHWK is presented as negative control since it showed no consistent inhibitory activity against the virus. Data is presented as means and standard deviations of two independent experiments.

the context of presentation in the M13 pIII protein. Then synthetic peptides were tested to check their ability to impair the infection of DENV2 in Vero cells. The synthetic peptides ELLASPW, SYQSHYY, and STSFWIT showed inhibitory activity against DENV2 infection in a plaque reduction test (Figure 5(b)). Some peptides that showed good in vitro binding to their target proteins did not show consistent inhibitory activity against the virus, that is, the peptide
SPSTHWK, and were considered negative for viral inhibition (Figure 5(b)). Since the peptides were incubated only during the adsorption period $\left(1 \mathrm{~h}\right.$ at $\left.37^{\circ} \mathrm{C}\right)$, it may be possible that the observed inhibition is due to interference in this initial step of the virus life cycle or due to virucidal activity. More experiments are required to elucidate the exact mechanism of action of these peptides. When the active peptides were incubated alone with Vero cells in the same conditions as 


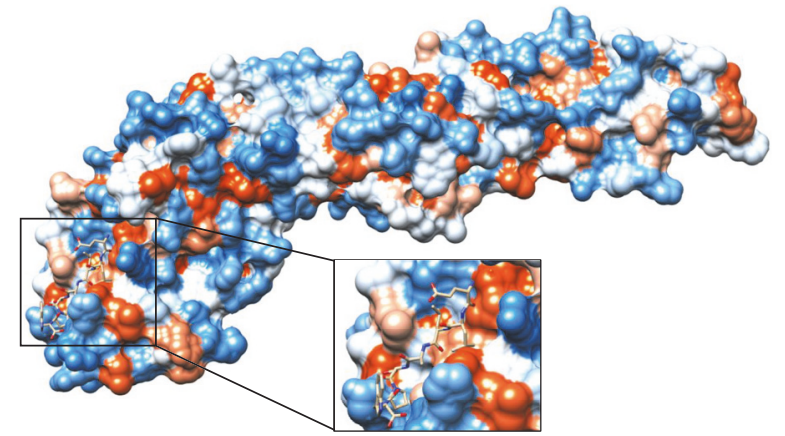

(a)

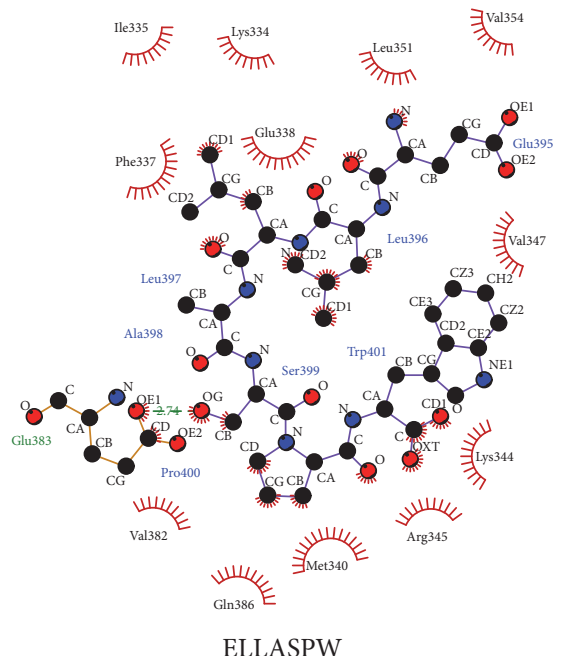

(c)

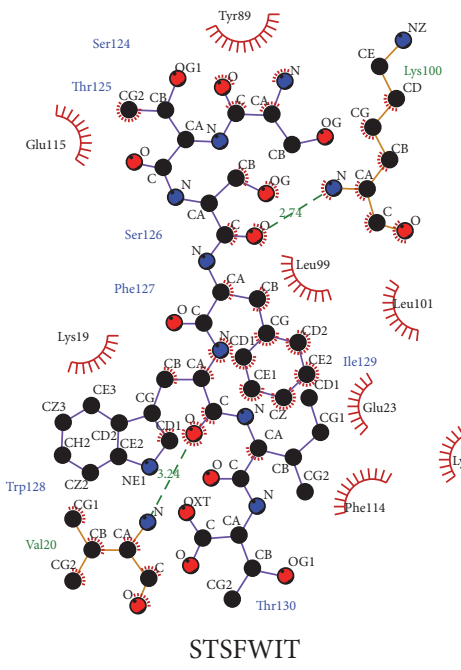

(d)

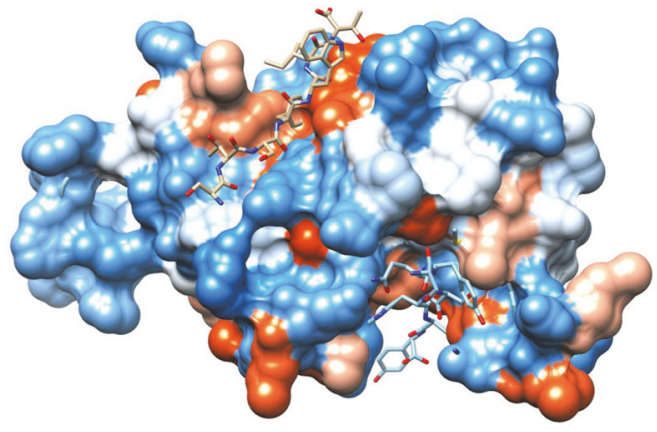

(b)

FIGURE 6: Probable binding modes of active peptides on their targets, as explored by molecular docking simulation. (a) Most favorable pose of peptide ELLASPW on the DENV2 envelope. Inset shows more details of putative binding site. (b) Most favorable poses of peptides STSFWIT and SYQSHYY on domain III. Peptide STSFWIT occupies the upper binding site of the molecule in this diagram. Proteins are depicted in their hydrophobicity surfaces, while peptides are in stick model. (c) Interaction diagram for ELLASPW-envelope. (d) Interaction diagram for STSFWIT-domain III. (e) Interaction diagram for SYQSHYY-domain III. Interaction diagrams were done in LigPlot+. Intermolecular hydrogen bonds are indicated by green dashed lines, while hydrophobic interactions are represented by arcs and radiating spokes. Pose figures were prepared using UCSF Chimera.

those of the viral infection, no sign of cytotoxicity was observed (Figure 5(a)).

The fact that some other peptides with good binding activity at the panning and ELISA tests did not inhibit the DENV2 infection is not surprising, as some of these small peptides might be binding to target regions that are not critical for their functions during binding and entry. It may be also possible that some of these peptides interact differently with their targets in their synthetic form compared with the case when they were presented in the context of the phage protein pIII.

In order to explore possible modes of binding of these peptides to their targets, we performed computational docking analysis using CABS-dock online server [25]. The algorithm pipeline followed by this software allows flexible, fully blind protein-peptide docking, finishing with all atom reconstruction and optimization of the best poses [41, 42]. The structures of the proteins used for the docking were either obtained from the database of protein structures (in the case of the DENV2 envelope protein, PDB 1OAN) or obtained in our lab by homology modeling (in the case of the DENV2 EDIII).

The docking simulations allowed prediction of possible poses for the binding of the active peptides to their target proteins. The best models predicted indicated a predominance of hydrophobic interactions over hydrogen bonds (Figure 6). Interestingly, the best pose predicted for the envelope glycoprotein and the corresponding peptide ELLASPW proposed a binding site also at the domain III of the protein (Figure 6). The suggested pose with the lowest interaction energy for the peptide ELLASPW on the envelope glycoprotein indicates hydrophobic interactions of the peptide with the protein residues I335, K334, L351, V354, F337, E338, V347, K344, R345, M340, Q386, and V382 and a hydrogen bond with E383. The suggested interactions of peptide STSFWIT with DIII include hydrophobic links with Y377, E403, L387, L389, 
K307, E311, K310, and F402 and hydrogen bonds with K388 and V308. Similarly, the best pose proposed for peptide SYQSHYY includes hydrophobic interactions with V382, M301, P336, E338, L294, K334, F337, I339, N355, M340, K295, V354, and R350 and hydrogen bond with K291.

Domain III of the dengue envelope has been shown to be involved in the initial steps of the binding of the virion to the receptor/attachment factors, particularly the amino acids in the sequence 380-IGVEPGQLKL-389 [13, 43, 44]. Interestingly, the best poses proposed by the simulation indicate some overlap with this region. The peptide STSFWIT appears to bind a region of DIII overlapping the 380-389 sequence, with three of its amino acids interacting with the peptide: L387, K388, and L389. The peptide ELLASPW does not appear to block or interfere with the receptor binding sequence, although two of its residues do participate in hydrophobic interactions with the peptide, E383 and Q386. The peptide SYQSHYY does not bind overlapping the receptor binding region, although one of its amino acids does interact with the peptide (V382). Interestingly, this last peptide also appears to bind very close to the sequence DKLQLK, interacting with the residues K291, Q293, and L294. This binding site may be relevant to the entry process as lysine 291 and lysine 295 were shown to be key for binding to the cellular GAGs [45]. Although best poses proposed here for the interactions between these peptides and their targets show favorable interaction energy profiles (data not shown), it should be also noted that, due to the complexities of modeling small peptides, there may be other valid binding sites. Further studies may be required to validate the proposed binding modes of the peptides and perform a rational, structure directed improvement of their biological activities.

The phage display technique has been used by several authors to search for interacting peptides, with the ultimate objective of developing new drugs against several viruses. Some examples include peptides described with activity against HIV [21, 46], Puumala virus [47], West Nile virus [48], Newcastle virus [49, 50], hepatitis C virus [51, 52], porcine reproductive and respiratory syndrome virus $[53,54]$, infectious bronchitis virus [55], hepatitis B virus [56], Mink enteritis virus [57], Japanese encephalitis virus [58], classical swine fever virus [59], and influenza virus [60].

Regarding peptidic inhibitors of dengue virus, three main strategies have been followed: (1) in silico designed peptides, (2) peptides mimicking the viral protein sequences, and (3) peptides obtained by panning against viral proteins. While the development of antiviral peptides against DENV has been dominated by reports using the first two approaches [6164], a few attempts have been made to make use of the versatile phage display technology to find new antivirals. The peptides identified using random peptide libraries include those reported by Panya et al., 2014 [65], which target the envelope hydrophobic pocket, and those reported by Chew et al., 2015 [66], which target the full DENV2 virion. The peptides reported here do not share homology with the ones found by these authors, although results may not be fully comparable as different targets and libraries were used.

\section{Conclusions}

In conclusion, we have used a novel strategy to identify inhibitors of the DENV2 infectivity and we have found three peptides out of a random peptide library, which are able to specifically bind viral envelope protein and inhibit infectivity in vitro, without showing toxicity to the cells. The binding modes suggested by blind docking simulations indicate that the interactions between these active peptides and their targets may be stabilized by hydrophobic interactions, providing information relevant to the future improvement of new antivirals against this important virus.

\section{Conflicts of Interest}

The authors declare that there are no conflicts of interest.

\section{Authors' Contributions}

Carolina de la Guardia participated in experimental work and drafting of the manuscript. Mario Quijada participated in the execution of the experimental work. Ricardo Lleonart participated in the design, experimental work, and manuscript preparation.

\section{Acknowledgments}

This work was partially supported by grants from Banco Interamericano de Desarrollo (Grant no. IND-JAL-02DENGUE), Secretaría Nacional de Ciencia, Tecnología e Innovación de Panamá (SENACYT), and Sistema Nacional de Investigación de Panamá (SNI). Authors are also grateful to Alejandro Llanes for revision of the manuscript and to researchers from ICGES, Panamá, for donation of viral DENV2 strain.

\section{References}

[1] S. Bhatt, P. W. Gething, O. J. Brady et al., "The global distribution and burden of dengue," Nature, vol. 496, no. 7446, pp. 504-507, 2013.

[2] O. J. Brady, P. W. Gething, S. Bhatt et al., "Refining the global spatial limits of dengue virus transmission by evidence-based consensus," PLoS Neglected Tropical Diseases, vol. 6, no. 8, Article ID e1760, 2012.

[3] WHO, Dengue and severe dengue, World Health organization, 2017, http://www.who.int/mediacentre/factsheets/fs117/en/.

[4] J. S. Mackenzie, D. J. Gubler, and L. R. Petersen, "Emerging flaviviruses: the spread and resurgence of Japanese encephalitis, West Nile and dengue viruses," Nature Medicine, vol. 10, no. 12, pp. S98-S109, 2004.

[5] J. P. Messina, O. J. Brady, T. W. Scott et al., "Global spread of dengue virus types: mapping the 70 year history," Trends in Microbiology, vol. 22, no. 3, pp. 138-146, 2014.

[6] D. A. Shroyer, "Aedes albopictus and arboviruses: a concise review of the literature," Journal of the American Mosquito Control Association, vol. 2, no. 4, pp. 424-428, 1986. 
[7] R. J. Kuhn, W. Zhang, M. G. Rossmann, S. V Pletnev et al., "Structure of dengue virus: implications for flavivirus organization, maturation, and fusion," Cell, vol. 108, no. 5, pp. 717-725, 2002.

[8] B. D. Lindenbach and C. M. Rice, "Flaviviridae: the viruses and their replication," Fields Virol, pp. 1101-1151, 2007.

[9] S. Bressanelli, K. Stiasny, S. L. Allison et al., "Structure of a flavivirus envelope glycoprotein in its low-pH-induced membrane fusion conformation," The EMBO Journal, vol. 23, no. 4, pp. 728-738, 2004.

[10] M. Liao and M. Kielian, "Domain III from class II fusion proteins functions as a dominant-negative inhibitor of virus membrane fusion," Journal of Cell Biology, vol. 171, no. 1, pp. 111120, 2005.

[11] D. Kato, S. Era, I. Watanabe et al., "Antiviral activity of chondroitin sulphate E targeting dengue virus envelope protein," Antiviral Research, vol. 88, no. 2, pp. 236-243, 2010.

[12] C. O. Nicholson, J. M. Costin, D. K. Rowe et al., "Viral entry inhibitors block dengue antibody-dependent enhancement in vitro," Antiviral Research, vol. 89, no. 1, pp. 71-74, 2011.

[13] F. A. Rey, F. X. Heinz, C. Mandl, C. Kunz, and S. C. Harrison, "The envelope glycoprotein from tick-borne encephalitis virus at 2 Å resolution," Nature, vol. 375, no. 6529, pp. 291-298, 1995.

[14] Y. Modis, S. Ogata, D. Clements, and S. C. Harrison, "A ligandbinding pocket in the dengue virus envelope glycoprotein," Proceedings of the National Academy of Sciences of the United States of America, vol. 100, no. 12, pp. 6986-6991, 2003.

[15] Y. Modis, S. Ogata, D. Clements, and S. C. Harrison, "Structure of the dengue virus envelope protein after membrane fusion," Nature, vol. 427, no. 6972, pp. 313-319, 2004.

[16] S. L. Allison, K. Stiasny, K. Stadler, C. W. Mandl, and F. X. Heinz, "Mapping of functional elements in the stem-anchor region of tick-borne encephalitis virus envelope protein E," Journal of Virology, vol. 73, no. 7, pp. 5605-5612, 1999.

[17] J. Hung, M. Hsieh, M. Young, C. Kao, C. King, and W. Chang, "An external loop region of domain III of dengue virus type 2 envelope protein is involved in serotype-specific binding to mosquito but not mammalian cells," Journal of Virology, vol. 78, no. 1, pp. 378-388, 2004.

[18] W. D. Crill and J. T. Roehrig, "Monoclonal antibodies that bind to domain III of dengue virus E glycoprotein are the most efficient blockers of virus adsorption to vero cells," Journal of Virology, vol. 75, no. 16, pp. 7769-7773, 2001.

[19] A. R. Precioso, R. Palacios, B. Thomé, G. Mondini, P. Braga, and J. Kalil, "Clinical evaluation strategies for a live attenuated tetravalent dengue vaccine," Vaccine, vol. 33, no. 50, pp. 71217125, 2015.

[20] "Weekly Epidemiological Record (WER)," vol. 91, no. 30, 349-364, 2016, http://www.who.int/wer/.

[21] M. Ferrer and S. C. Harrison, "Peptide ligands to human immunodeficiency virus type 1 gp120 identified from phage display libraries," Journal of Virology, vol. 73, no. 7, pp. 57955802, 1999.

[22] E. Koivunen, W. Arap, D. Rajotte, J. Lahdenranta, and R. Pasqualini, "Identification of receptor ligands with phage display peptide libraries," Journal of Nuclear Medicine, vol. 40, no. 10, pp. 883-888, 1999.

[23] A. M. Waterhouse, J. B. Procter, D. M. A. Martin, M. Clamp, and G. J. Barton, "Jalview Version 2-A multiple sequence alignment editor and analysis workbench," Bioinformatics, vol. 25, no. 9, pp. 1189-1191, 2009.
[24] J. Yang, R. Yan, A. Roy, D. Xu, J. Poisson, and Y. Zhang, "The I-TASSER Suite: protein structure and function prediction," Nature Methods, vol. 12, no. 1, pp. 7-8, 2015.

[25] M. Kurcinski, M. Jamroz, M. Blaszczyk, A. Kolinski, and S. Kmiecik, "CABS-dock web server for the flexible docking of peptides to proteins without prior knowledge of the binding site," Nucleic Acids Research, vol. 43, no. 1, pp. W419-W424, 2015.

[26] R. A. Laskowski and M. B. Swindells, "LigPlot+: multiple ligandprotein interaction diagrams for drug discovery," Journal of Chemical Information and Modeling, vol. 51, no. 10, pp. 27782786, 2011.

[27] Y. Modis, S. Ogata, D. Clements, and S. C. Harrison, "Variable surface epitopes in the crystal structure of dengue virus type 3 envelope glycoprotein," Journal of Virology, vol. 79, no. 2, pp. 1223-1231, 2005.

[28] Y. Zhang, W. Zhang, S. Ogata et al., "Conformational changes of the flavivirus E glycoprotein," Structure, vol. 12, no. 9, pp. 16071618, 2004.

[29] J. T. Roehrig, R. A. Bolin, and R. G. Kelly, "Monoclonal antibody mapping of the envelope glycoprotein of the dengue 2 virus, Jamaica," Virology, vol. 246, no. 2, pp. 317-328, 1998.

[30] P. Thullier, P. Lafaye, F. Mégret, V. Deubel, A. Jouan, and J. C. Mazié, "A recombinant Fab neutralizes dengue virus in vitro," Journal of Biotechnology, vol. 69, no. 2-3, pp. 183-190, 1999.

[31] A. P. J. Middelberg, "Preparative protein refolding," Trends in Biotechnology, vol. 20, no. 10, pp. 437-443, 2002.

[32] M.-W. Chiu and Y.-L. Yang, "Blocking the dengue virus 2 infections on BHK-21 cells with purified recombinant dengue virus 2 E protein expressed in Escherichia coli," Biochemical and Biophysical Research Communications, vol. 309, no. 3, pp. 672678, 2003.

[33] J. J. H. Chu, R. Rajamanonmani, J. Li, R. Bhuvananakantham, J. Lescar, and M.-L. Ng, "Inhibition of West Nile virus entry by using a recombinant domain III from the envelope glycoprotein," Journal of General Virology, vol. 86, part 2, pp. 405-412, 2005.

[34] Z.-S. Zhang, Y.-S. Yan, Y.-W. Weng et al., "High-level expression of recombinant dengue virus type 2 envelope domain III protein and induction of neutralizing antibodies in BALB/C mice," Journal of Virological Methods, vol. 143, no. 2, pp. 125-131, 2007.

[35] J. F. L. Chin, J. J. H. Chu, and M. L. Ng, "The envelope glycoprotein domain III of dengue virus serotypes 1 and 2 inhibit virus entry," Microbes and Infection, vol. 9, no. 1, pp. 16, 2007.

[36] B. He, G. Chai, Y. Duan et al., "BDB: biopanning data bank," Nucleic Acids Research, vol. 44, pp. D1127-D1132, 2016.

[37] M. Ploss, S. J. Facey, C. Bruhn et al., "Selection of peptides binding to metallic borides by screening M13 phage display libraries," BMC Biotechnology, vol. 14, article no. 12, 2014.

[38] S. Swaminathan and Y. Cui, "Recognition of epoxy with phage displayed peptides," Materials Science and Engineering C, vol. 33, no. 5, pp. 3082-3084, 2013.

[39] N. Ojeda, C. Cárdenas, F. Guzmán, and S. H. Marshall, “Chemical synthesis and in vitro evaluation of a phage display-derived peptide active against infectious salmon anemia virus," Applied and Environmental Microbiology, vol. 82, no. 8, pp. 2563-2571, 2016.

[40] S. Swaminathan and Y. Cui, "Recognition of poly(dimethylsiloxane) with phage displayed peptides," RSC Advances, vol. 2, no. 33, pp. 12724-12727, 2012. 
[41] M. Blaszczyk, M. Kurcinski, M. Kouza et al., "Modeling of protein-peptide interactions using the CABS-dock web server for binding site search and flexible docking," Methods, vol. 93, pp. 72-83, 2016.

[42] M. P. Ciemny, M. Kurcinski, K. J. Kozak, A. Kolinski, and S. Kmiecik, "Highly flexible protein-peptide docking using CABSdock," in Modeling Peptide-Protein Interactions, vol. 1561 of Methods in Molecular Biology, pp. 69-94, 2017.

[43] S. Mukhopadhyay, R. J. Kuhn, and M. G. Rossmann, "A structural perspective of the Flavivirus life cycle," Nature Reviews Microbiology, vol. 3, no. 1, pp. 13-22, 2005.

[44] S. M. Erb, S. Butrapet, K. J. Moss et al., "Domain-III FG loop of the dengue virus type 2 envelope protein is important for infection of mammalian cells and Aedes aegypti mosquitoes," Virology, vol. 406, no. 2, pp. 328-335, 2010.

[45] D. Watterson, B. Kobe, and P. R. Young, "Residues in domain III of the dengue virus envelope glycoprotein involved in cell-surface glycosaminoglycan binding," Journal of General Virology, vol. 93, no. 1, pp. 72-82, 2012.

[46] B. D. Welch, J. N. Francis, J. S. Redman et al., "Design of a potent D-peptide HIV-1 entry inhibitor with a strong barrier to resistance," Journal of Virology, vol. 84, no. 21, pp. 11235-11244, 2010.

[47] T. Heiskanen, A. Lundkvist, A. Vaheri, and H. Lankinen, "Phage-displayed peptide targeting on the Puumala hantavirus neutralization site," Journal of Virology, vol. 71, no. 5, pp. 38793885, 1997.

[48] F. Bai, T. Town, D. Pradhan et al., "Antiviral peptides targeting the West Nile virus envelope protein," Journal of Virology, vol. 81, no. 4, pp. 2047-2055, 2007.

[49] M. Ozawa, K. Ohashi, and M. Onuma, "Identification and characterization of peptides binding to Newcastle disease virus by phage display," Journal of Veterinary Medical Science, vol. 67, no. 12, pp. 1237-1241, 2005.

[50] S. L. Chia, W. S. Tan, K. Shaari, N. Abdul Rahman, K. Yusoff, and S. D. Satyanarayanajois, "Structural analysis of peptides that interact with Newcastle disease virus," Peptides, vol. 27, no. 6, pp. 1217-1225, 2006.

[51] H. W. Hong, S. W. Lee, and H. Myung, "Selection of peptides binding to HCV E2 and inhibiting viral infectivity," Journal of Microbiology and Biotechnology, vol. 20, no. 12, pp. 1769-1771, 2010.

[52] X. Lü, M. Yao, J.-M. Zhang et al., "Identification of peptides that bind hepatitis $\mathrm{C}$ virus envelope protein $\mathrm{E} 2$ and inhibit viral cellular entry from a phage-display peptide library," International Journal of Molecular Medicine, vol. 33, no. 5, pp. 1312-1318, 2014.

[53] K. Liu, X. Feng, Z. Ma et al., "Antiviral activity of phage display selected peptides against Porcine reproductive and respiratory syndrome virus in vitro," Virology, vol. 432, no. 1, pp. 73-80, 2012.

[54] H. Wang, R. Liu, J. Cui et al., "Characterization and utility of phages bearing peptides with affinity to porcine reproductive and respiratory syndrome virus nsp7 protein," Journal of Virological Methods, vol. 222, pp. 231-241, 2015.

[55] B. Peng, H. Chen, Y. Tan, M. Jin, H. Chen, and A. Guo, "Identification of one peptide which inhibited infectivity of avian infectious bronchitis virus in vitro," Science in China, Series C: Life Sciences, vol. 49, no. 2, pp. 158-163, 2006.

[56] K. L. Ho, K. Yusoff, H. F. Seow, and W. S. Tan, "Selection of high affinity ligands to hepatitis B core antigen from a phagedisplayed cyclic peptide library," Journal of Medical Virology, vol. 69, no. 1, pp. 27-32, 2003.
[57] Q. Zhang, Y. Wang, Q. Ji et al., "Selection of antiviral peptides against mink enteritis virus using a phage display peptide library," Current Microbiology, vol. 66, no. 4, pp. 379-384, 2013.

[58] X. Zu, Y. Liu, S. Wang et al., "Peptide inhibitor of Japanese encephalitis virus infection targeting envelope protein domain III," Antiviral Research, vol. 104, no. 1, pp. 7-14, 2014.

[59] L. Yin, Y. Luo, B. Liang et al., "Specific ligands for classical swine fever virus screened from landscape phage display library," Antiviral Research, vol. 109, no. 1, pp. 68-71, 2014.

[60] T. Matsubara, A. Onishi, D. Yamaguchi, and T. Sato, "Heptapeptide ligands against receptor-binding sites of influenza hemagglutinin toward anti-influenza therapy," Bioorganic and Medicinal Chemistry, vol. 24, no. 5, pp. 1106-1114, 2016.

[61] Y. M. Hrobowski, R. F. Garry, and S. F. Michael, "Peptide inhibitors of dengue virus and West Nile virus infectivity," Virology Journal, vol. 2, article 49, 2005.

[62] A. G. Schmidt, P. L. Yang, and S. C. Harrison, "Peptide inhibitors of dengue-virus entry target a late-stage fusion intermediate," PLOS Pathogens, vol. 6, no. 4, Article ID e1000851, 2010.

[63] A. G. Schmidt, P. L. Yang, and S. C. Harrison, "Peptide inhibitors of flavivirus entry derived from the E protein stem," Journal of Virology, vol. 84, no. 24, pp. 12549-12554, 2010.

[64] A. Panya, N. Sawasdee, M. Junking, C. Srisawat, K. Choowongkomon, and P.-T. Yenchitsomanus, "A Peptide Inhibitor Derived from the Conserved Ectodomain Region of DENV Membrane (M) Protein with Activity Against Dengue Virus Infection," Chemical Biology and Drug Design, vol. 86, no. 5, pp. 1093-1104, 2015.

[65] A. Panya, K. Bangphoomi, K. Choowongkomon, and P.-T. Yenchitsomanus, "Peptide inhibitors against dengue virus infection," Chemical Biology and Drug Design, vol. 84, no. 2, pp. 148157, 2014.

[66] M.-F. Chew, H.-W. Tham, M. Rajik, and S. H. Sharifah, "Antidengue virus serotype 2 activity and mode of action of a novel peptide," Journal of Applied Microbiology, vol. 119, no. 4, pp. 1170-1180, 2015. 

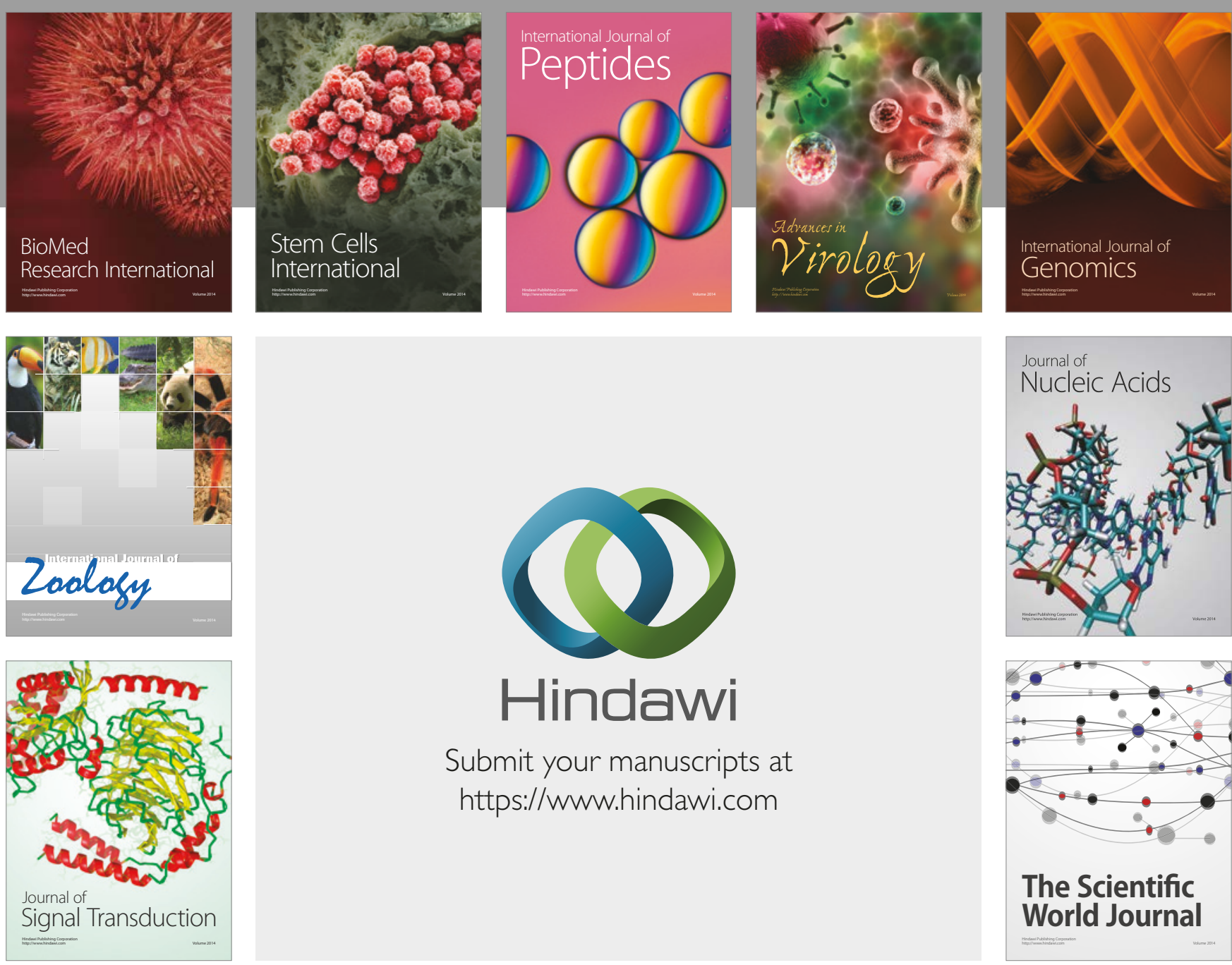

Submit your manuscripts at

https://www.hindawi.com
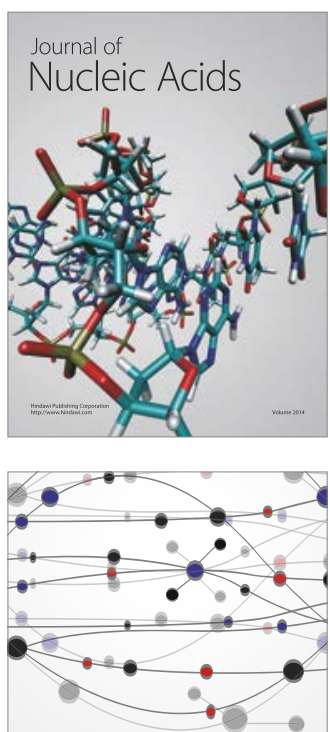

The Scientific World Journal

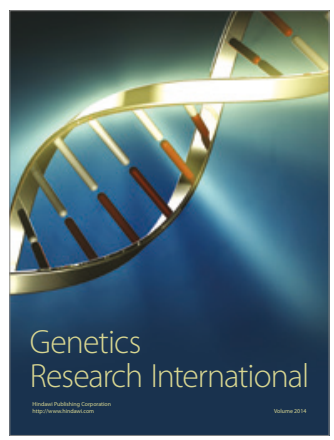

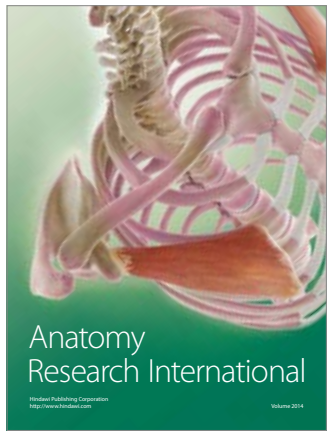

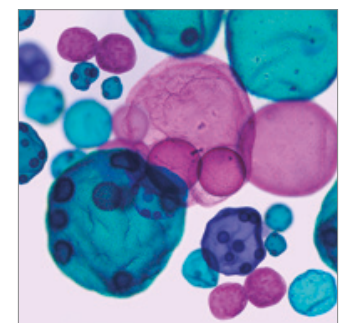

International Journal of Microbiology
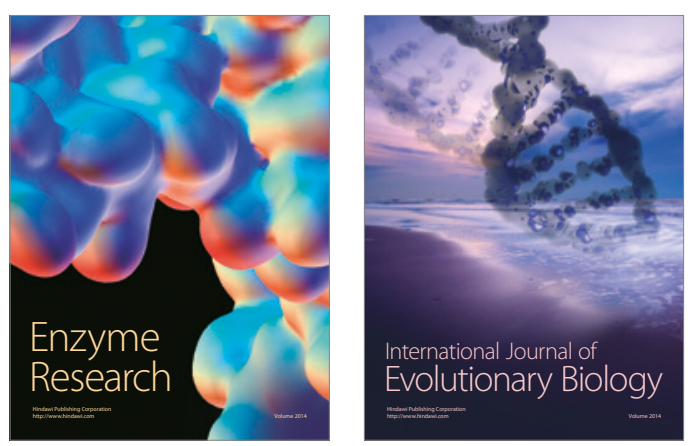
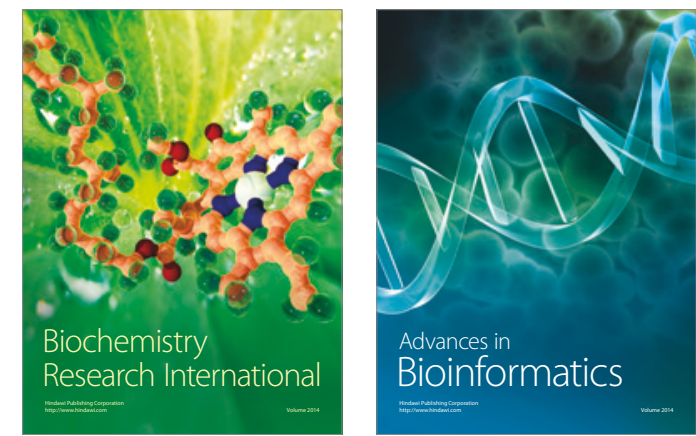

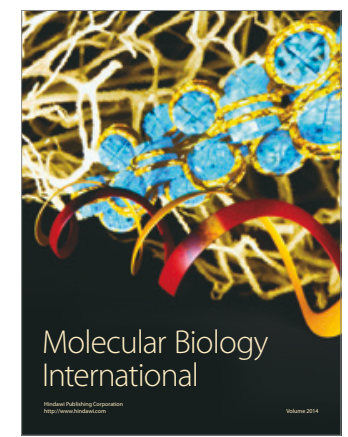

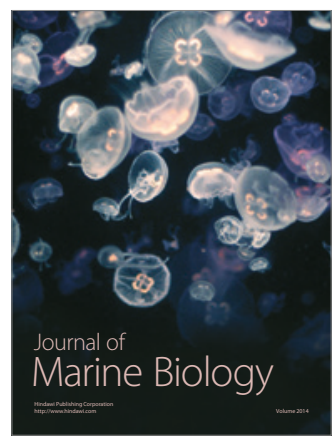

